\title{
ABOUT GLOBAL SOLUTION OF NONHOMOGENEOUS NEUTRAL PARTIAL DIFFERENTIAL EQUATION WITH DEVIATING ARGUMENT IN THE TIME VARIABLE
}

\author{
ANATOLY M. SAMOILENKO AND LIDIYA M. SERGEEVA
}

Received 24 September, 2018

\begin{abstract}
One class of nonhomogeneous neutral partial differential equations with deviating argument in the time variable is investigated. We find conditions under which it is possible to construct global solutions of these equations. We describe the structure of these solutions and their construction algorithm and we prove the theorem about substantiation of this method.
\end{abstract}

2010 Mathematics Subject Classification: 39A06; 39A14; 34K40

Keywords: global solution, deviating argument, a complete normed space, contraction mapping principle

\section{INTRODUCTION}

Very often neutral differential equations are used in mathematical models of many problems in natural science to describe processes the velocity of which at the given time depends on the state and velocity at the previous moments. For example, such equations arise in the simulation of solid state body motion controllers in systems with feedback, in the transmission of lossless current, in the study of self-oscillations in a long circuit with a tunnel diode, in some problems of control theory, etc.

We should mention recent works on finding solutions for such equations and studying their properties, including articles J. Banaś and I.J. Cabrera [1], D.J. Khusainov, J. Diblik, E.I. Kuzmich [6], W.G. El-Sayed [3], S. Karimi Vanani, A. Aminataei [14].

It's important to study the conditions of existence of the global solutions for neutral differential equations. Works $[13,15,16]$ are devoted to solve this problem. In some of these papers, the theorems of uniqueness and continuity of solutions are obtained on a small segment at first, and then the method of steps is applied. The theorems in [9] allow us to reduce the question of a continuity (or uniqueness) of solutions of a neutral differential-functional equation to the study of behavior of solutions of scalar differential inequalities. 
Some works should be noted, related to the existence and uniqueness of the solutions of boundary and initial boundary-value problems for neutral equations, in particular, there are works of G.A. Kamenskij, A.D. Myshkis, A.L. Skubachevskij [4], M.E. Drakhlin [2], O.I. Kiun [7], N.G. Kazakova [5], L.A. Minazhdinova [8].

\section{THE MAIN RESEARCH RESULTS}

In this paper an algorithm for constructing a global solution for a nonhomogeneous neutral partial differential equation with deviating argument in the time variable is described, and the conditions for its existence are given.

Consider the equation

$$
u_{t}(x, t)=p(t) u_{x x}(x, t+\mu)+r(t) u_{t}(x, t+\mu)+q(x, t),(x, t) \in Q,
$$

with zero boundary conditions

$$
u(0, t)=u(l, t)=0, t \in \mathbb{R},
$$

where $Q=\{(x, t): 0<x<l, t \in \mathbb{R}\}, p(t), r(t)$ are continuous functions in $\mathbb{R}$, and the deviation of $\mu$ is small enough.

2.1. Determining the structure of solution for problem (2.1), (2.2)

Previously in [11], it has been found that the corresponding homogeneous problem

$$
u_{t}(x, t)=p(t) u_{x x}(x, t+\mu)+r(t) u_{t}(x, t+\mu)
$$

has its eigenfunctions and eigenvalues respectively

$$
X_{k}(x)=\sin \frac{k \pi x}{l}, \lambda_{k}=\left(\frac{\pi k}{l}\right)^{2}, k=1, \ldots, n,
$$

with some $n \geq 1$.

Consider the nonhomogeneous equation (2.1). We construct a global solution $u(x, t)$ of the problem $(2.1),(2.2)$ as a sum

$$
u(x, t)=\sum_{k=1}^{n} X_{k}(x) T_{k}(t) .
$$

Obviously, the boundary conditions are satisfied.

It is assumed that the function $q(x, t)$ can be represented as a sum of the first $n$ terms of the Fourier series:

$$
q(x, t)=\sum_{k=1}^{n} q_{k}(t) \sin \frac{k \pi x}{l}, \quad q_{k}(t)=\frac{2}{l} \int_{0}^{l} q(\xi, t) \sin \frac{k \pi \xi}{l} d \xi .
$$

Putting (2.3) in equation (2.1), and taking into account (2.4), we have:

$$
\sum_{k=1}^{n}\left[T_{k}^{\prime}(t)-r(t) T_{k}^{\prime}(t+\mu)+\lambda_{k} p(t) T_{k}(t+\mu)-q_{k}(t)\right] \sin \frac{k \pi x}{l}=0 .
$$


This equation is satisfied if all the expansion coefficients equal to zero, that is

$$
T_{k}^{\prime}(t)-r(t) T_{k}^{\prime}(t+\mu)+\lambda_{k} p(t) T_{k}(t+\mu)-q_{k}(t)=0, k=1, \ldots, n .
$$

In work [12] it was shown, that for this equation we can construct corresponding equation without deviating argument, all the solutions of which will be global solutions of the equation (2.5). This equation has a form

$$
T_{k}^{\prime}(t)+\bar{p}_{k}(t) T_{k}(t)-\bar{q}_{k}(t)=0, k=1, \ldots, n .
$$

We can define $\bar{p}(t)$ and $\bar{q}(t)$ according to the algorithm defined in [10]. The general solution of equation (2.6) is determined by Cauchy formula

$$
T_{k}(t)=T_{k, 0} e^{-\int_{t_{0}}^{t} \bar{p}_{k}(s) d s}+\int_{t_{0}}^{t} \bar{q}_{k}(\tau) e^{-\int_{\tau}^{t} \bar{p}_{k}(s) d s} d \tau,
$$

where

$$
T_{k, 0}=T_{k}\left(t_{0}\right), \quad t, t_{0} \in \mathbb{R} .
$$

This function satisfies equation (2.5) if

$$
\begin{aligned}
T_{k}^{\prime}(t)= & -\bar{p}_{k}(t)\left[T_{k, 0} e^{-\int_{t_{0}}^{t} \bar{p}_{k}(s) d s}+\int_{t_{0}}^{t} \bar{q}_{k}(\tau) e^{-\int_{\tau}^{t} \bar{p}_{k}(s) d s} d \tau\right]+\bar{q}_{k}(t) \\
= & r(t)\left[-\bar{p}_{k}(t+\mu)\left[T_{k, 0} e^{-\int_{t_{0}}^{t+\mu} \bar{p}_{k}(s) d s}+\int_{t_{0}}^{t+\mu} \bar{q}_{k}(\tau) e^{-\int_{\tau}^{t+\mu} \bar{p}_{k}(s) d s} d \tau\right]+\bar{q}_{k}(t+\mu)\right] \\
& -\lambda_{k} p(t)\left[T_{k, 0} e^{-\int_{t_{0}}^{t+\mu} \bar{p}_{k}(s) d s}+\int_{t_{0}}^{t+\mu} \bar{q}_{k}(\tau) e^{-\int_{\tau}^{t+\mu} \bar{p}_{k}(s) d s} d \tau\right]+q_{k}(t), \quad t \in \mathbb{R} . \quad \text { (2.7) }
\end{aligned}
$$

Putting in (2.7) $T_{k, 0}=0$, we have

$$
\begin{aligned}
& -\bar{p}_{k}(t)\left[\int_{t_{0}}^{t} \bar{q}_{k}(\tau) e^{-\int_{\tau}^{t} \bar{p}_{k}(s) d s} d \tau\right]+\bar{q}_{k}(t) \\
& =-\left(r(t) \bar{p}_{k}(t+\mu)+\lambda_{k} p(t)\right)\left[\int_{t_{0}}^{t+\mu} \bar{q}_{k}(\tau) e^{-\int_{\tau}^{t+\mu} \bar{p}_{k}(s) d s} d \tau\right] \\
& +r(t) \bar{q}_{k}(t+\mu)+q_{k}(t), \quad t \in \mathbb{R} .
\end{aligned}
$$

Taking into account (2.7) and (2.8), we obtain

$$
\bar{p}_{k}(t) e^{-\int_{t_{0}}^{t} \bar{p}_{k}(s) d s}=\left(r(t) \bar{p}_{k}(t+\mu)+\lambda_{k} p(t)\right) e^{-\int_{t_{0}}^{t+\mu} \bar{p}_{k}(s) d s},
$$


where we receive

$$
\bar{p}_{k}(t)=\left(r(t) \bar{p}_{k}(t+\mu)+\lambda_{k} p(t)\right) e^{\int_{t+\mu}^{t} \bar{p}_{k}(s) d s}, k \leq n, t \in \mathbb{R} .
$$

Substituting (2.9) in (2.8):

$$
\begin{aligned}
& -\left(r(t) \bar{p}_{k}(t+\mu)+\lambda_{k} p(t)\right) \int_{t_{0}}^{t} \bar{q}_{k}(\tau) e^{\int_{t+\mu}^{\tau} \bar{p}_{k}(s) d s} d \tau+\bar{q}_{k}(t) \\
& =-\left(r(t) \bar{p}_{k}(t+\mu)+\lambda_{k} p(t)\right) \int_{t_{0}}^{t+\mu} \bar{q}_{k}(\tau) e^{t+\mu} \int_{k}^{\tau}(s) d s \\
& \bar{q}_{k}(t)=q_{k}(t)+r(t) \bar{q}_{k}(t+\mu)+\left(r(t) \bar{p}_{k}(t+\mu)\right. \\
& \left.+\lambda_{k} p(t)\right) \int_{t+\mu}^{t} \bar{q}_{k}(\tau) e^{\int_{t+\mu}^{\tau} \bar{p}_{k}(s) d s} d \tau, k \leq n, \quad t \in \mathbb{R} .
\end{aligned}
$$

Then the solution $T_{k}(t)$ of equation (2.6) can be written as

$$
T_{k}(t)=c_{k} e^{-\int_{0}^{t} \bar{p}_{k}(s) d s}+\int_{0}^{t} \bar{q}_{k}(\tau) e^{-\int_{\tau}^{t} \bar{p}_{k}(s) d s} d \tau,
$$

where $c_{k}$ are arbitrary constants, $t_{0}=0$. And we find $\bar{p}_{k}(t)$ and $\bar{q}_{k}(t)$ using equations (2.9), (2.10).

So, if all the solutions of equation (2.6) are global solutions of equation (2.5), then function $\bar{p}$ satisfies equation (2.9), and function $\bar{q}$ satisfies equation (2.10). The existence of solutions for equations (2.9) and (2.10) is a necessary and sufficient condition to ensure that all solutions for equation (2.6) are global solutions for equation (2.5).

We found conditions under which the solution of equation (2.6) is the global solution of equation (2.5). The following theorem is the main result of our research.

Theorem 1. Assume functions $p, r$ and $q$ satisfy the conditions imposed above, moreover

$$
|p(t)|<\beta,|r(t)|<\gamma, \quad \beta, \gamma=\text { const }, \quad t \in \mathbb{R},
$$

and also the following inequality holds

$$
\gamma+\left(\gamma+\lambda_{n} \beta|\mu|\right) e<1, \quad \gamma<\frac{1}{e},
$$

where $n$ is an integer part of the number $\frac{l}{\pi} \sqrt{\frac{1-\gamma e}{e \beta|\mu|}}$.

Then the global solution exists in the form (2.3) for the problem (2.1), (2.2). 
Proof of Theorem 1. Consider the equation (2.9). The solution of this equation is a continuous function. We find conditions under which this equation has a unique solution, using the contraction mapping principle.

Let's define an operator $\left(S \bar{p}_{k}\right)(t)$ for function $\bar{p}_{k}$, which is continuous in $\mathbb{R}$.

$$
\left(S \bar{p}_{k}\right)(t)=\left(r(t) \bar{p}_{k}(t+\mu)+\lambda_{k} p(t)\right) e^{\int_{t+\mu}^{t} \bar{p}_{k}(s) d s} .
$$

We search solution in the space $C(m)$. Let $\left\|\bar{p}_{k}\right\|_{0}=\sup _{t \in \mathbb{R}}\left|\bar{p}_{k}(t)\right| \leq m$. Then the next estimation for the $\left(S \bar{p}_{k}\right)(t)$ is true

$$
\left\|S \bar{p}_{k}\right\|_{0}=\sup _{t \in \mathbb{R}}\left|\left(r(t) \bar{p}_{k}(t+\mu)+\lambda_{k} p(t)\right) e^{\int_{t+\mu}^{t} \bar{p}(s) d s}\right| \leq(\gamma m+\lambda \beta) e^{|\mu| m} .
$$

If inequality

$$
\left(\gamma m+\lambda_{k} \beta\right) e^{|\mu| m} \leq m
$$

is satisfied, then the operator $\left(S \bar{p}_{k}\right)(t)$ reflects the space $C(m)$ to itself.

We estimate the difference $\left(S \bar{p}_{k, 1}\right)(t)-\left(S \bar{p}_{k, 2}\right)(t)$ :

$$
\begin{aligned}
\left|\left(S \bar{p}_{k, 1}\right)(t)-\left(S \bar{p}_{k, 2}\right)(t)\right|= & \mid r(t)\left(\bar{p}_{k, 1}(t+\mu) e^{\int_{t+\mu}^{t} \bar{p}_{k, 1}(s) d s}\right. \\
& \left.-\bar{p}_{k, 2}(t+\mu) e^{\int_{t+\mu}^{t} \bar{p}_{k, 2}(s) d s}\right) \\
& +\lambda_{k} p(t)\left(e^{\int_{t+\mu}^{t} \bar{p}_{k, 1}(s) d s}-e^{\int_{t+\mu}^{t} \bar{p}_{k, 2}(s) d s}\right) \mid \\
= & \mid r(t)\left(\bar{p}_{k, 1}(t+\mu)\left(e^{\int_{t+\mu}^{t} \bar{p}_{k, 1}(s) d s}-1\right)+\bar{p}_{k, 1}(t+\mu)\right. \\
& \left.-\bar{p}_{k, 2}(t+\mu)\left(e^{\int_{t+\mu}^{t} \bar{p}_{k, 2}(s) d s}-1\right)-\bar{p}_{k, 2}(t+\mu)\right) \\
& +\lambda_{k} p(t)\left(e^{\int_{t+\mu}^{t} \bar{p}_{k, 1}(s) d s}-e^{\int_{t+\mu}^{t} \bar{p}_{k, 2}(s) d s}\right) \mid \\
\leq & \left(\gamma+\lambda_{k} \beta|\mu|\right) e^{|\mu| m}\left\|\bar{p}_{k, 1}-\bar{p}_{k, 2}\right\|_{0} .
\end{aligned}
$$

Thus, when the inequality

$$
\left(\gamma+\lambda_{k} \beta|\mu|\right) e^{|\mu| m}<1
$$

is satisfied, then $\left(S \bar{p}_{k}\right)(t)$ is the contraction operator in the space $C(m)$.

We suppose conditions (2.13) and (2.14) are justified simultaneously. Let the following estimation be true

$$
\left(\gamma+\lambda_{k} \beta|\mu|\right) e<1
$$

Then the equation

$$
\left(\gamma m+\lambda_{k} \beta\right) e^{|\mu| m}=m
$$

has the solutions, for which the next condition is valid

$$
\frac{\left(\gamma+\lambda_{k} \beta|\mu|\right) m}{\gamma m+\lambda_{k} \beta}<1
$$


But the operator $\left(S \bar{p}_{k}\right)(t)$ is a contraction operator and reflects the space $C(m)$ to itself only for

$$
m<\frac{1}{|\mu|}
$$

Thus, if this condition is satisfied, then the space $C(m)$ is a complete normed space and the operator $S$ has a single fixed point in this space, so the solution of equation (2.9) exists and it is unique.

Consider the equation (2.10). Without loss of generality, we assume that $|q(x, t)| \leq \frac{1}{2}$.

We define the operator $S_{1}$ in the space $C(M)$ of the functions $\bar{q}_{k}$, which are defined and continuous in $\mathbb{R}$, such that

$$
\left\|\bar{q}_{k}\right\|_{0}=\sup _{t \in \mathbb{R}}\left|\bar{q}_{k}(t)\right| \leq M .
$$

$\left(S_{1} \bar{q}_{k}\right)(t)$ is continuous function in $\mathbb{R}$, and the next estimates are carried

$$
\begin{aligned}
\left\|S_{1} \bar{q}_{k}\right\|_{0} & \leq 1+\gamma M+\left(\gamma m+\lambda_{k} \beta\right)|\mu| M e^{|\mu| m}, \\
\left|\left(S \bar{q}_{k, 1}\right)(t)-\left(S \bar{q}_{k, 2}\right)(t)\right| & \leq \gamma+\left(\gamma m+\lambda_{k} \beta\right)|\mu| e^{|\mu| m}\left\|\bar{q}_{k, 1}-\bar{q}_{k, 2}\right\|_{0},
\end{aligned}
$$

where $\bar{q}_{k, 1}, \bar{q}_{k, 2}$ are arbitrary functions in $C(M)$.

The condition $\gamma+\left(\gamma+\lambda_{k} \beta|\mu|\right) e<1$ is justified due to the (2.12), so if

$$
M \geq \frac{1}{1-\gamma-\left(\gamma m+\lambda_{k} \beta\right)|\mu| e^{|\mu| m}}
$$

then operator $S_{1}$ is a contraction operator and reflects the space $C(M)$ to itself. The space $C(M)$ is a complete normed space. And it's enough that the operator $S_{1}$ has a single fixed point in the space $C(M)$. This point is a single solution of equation (2.10) in $C(M)$.

Let us find a range of values for $k$. Since $\lambda_{k}=\left(\frac{\pi k}{l}\right)^{2}$, we can find an approximate estimate for parameter $k$ from condition (2.15).

$$
k<\frac{l}{\pi} \sqrt{\frac{1-\gamma e}{e \beta|\mu|}} .
$$

Hence, $k \in[1, n]$, where $n$ is an integer part of $\frac{l}{\pi} \sqrt{\frac{1-\gamma e}{e \beta|\mu|}}$.

Therefore, the global solution of equation (2.5) has a form (2.11), where $\bar{p}_{k}(t)$ and $\bar{q}_{k}(t)$ are the solutions of equations (2.9), (2.10), and $k$ is determined by the formula (2.16).

\subsection{Solving the equations (2.9), (2.10)}

We solve the equations (2.9), (2.10) for $\bar{p}_{k}(t)$ and $\bar{q}_{k}(t)$ by the method of successive approximations. 
For initial approximation we take such:

$$
\bar{p}_{k}^{(0)}(t)=\lambda_{k} p(t), \bar{q}_{k}^{(0)}(t)=q(t) .
$$

Then

$$
\begin{aligned}
\bar{p}_{k}^{(\eta)}(t)= & \left(r(t) \bar{p}_{k}^{(\eta-1)}(t+\mu)+\lambda_{k} p(t)\right) e^{\int_{t+\mu}^{t} \bar{p}_{k}^{(\eta-1)}(\tau) d \tau}, \quad \eta=1,2, \ldots, \\
\bar{q}_{k}^{(v)}(t)= & q_{k}(t)+r(t) \bar{q}_{k}^{(\nu-1)}(t+\mu)+\left(r(t) \bar{p}_{k}^{(\eta)}(t+\mu)+\lambda_{k} p(t)\right) \\
& \times \int_{t+\mu}^{t} \bar{q}_{k}^{(\nu-1)}(\tau) e^{\int_{t+\mu}^{\tau} \bar{p}_{k}^{(\eta)}(s) d s} d \tau, \quad v=1,2, \ldots, \quad k=\overline{1, n}, \quad t \in \mathbb{R} .
\end{aligned}
$$

These sequences are uniformly convergent in $\mathbb{R}$ due to the conditions of the theorem, so

$$
\bar{p}_{k}=\lim _{\eta \rightarrow \infty} \bar{p}_{k}^{(\eta)}, \bar{q}_{k}=\lim _{\nu \rightarrow \infty} \bar{q}_{k}^{(v)} .
$$

Let us write the first approximation for $\bar{p}_{k}$ in explicit form

$$
\bar{p}_{k}^{(1)}(t)=(r(t) p(t+\mu)+p(t)) \lambda_{k} e^{\lambda_{k} \int_{t+\mu}^{t} p(s) d s} .
$$

Taking into account conditions of Theorem 1, we can obtain the following estimate:

$$
\left|\bar{p}_{k}^{(1)}(t)-\bar{p}_{k}^{(0)}(t)\right| \leq \lambda_{k} \beta\left((\gamma+1) e^{|\mu| \beta \lambda_{k}}-1\right) .
$$

According to estimates by the method of successive approximations such an assessment takes place for each next approximation

$$
\left|\bar{p}_{k}^{(j)}-\bar{p}_{k}\right| \leq \frac{\alpha^{j}\left|\bar{p}_{k}^{(1)}-\bar{p}_{k}^{(0)}\right|}{1-\alpha} .
$$

In our case $\alpha=\left(\gamma+\lambda_{k} \beta|\mu|\right) e$, then we obtain for $j=\eta$ :

$$
\left|\bar{p}_{k}^{(\eta)}-\bar{p}_{k}\right| \leq \frac{\alpha^{\eta} \lambda_{k} \beta\left|(\gamma+1) e^{|\mu| \beta \lambda_{k}}-1\right|}{1-\alpha} .
$$

Let us write the first approximation for $\bar{q}_{k}$ in explicit form

$$
\begin{aligned}
\bar{q}_{k}^{(1)}(t)= & q(t)+r(t) q(t+\mu)+\left(r(t) \bar{p}_{k}^{(\eta)}(t+\mu)+\lambda_{k} p(t)\right) \\
& \times \int_{t+\mu}^{t} q(\tau) e^{\int_{t+\mu}^{\tau} \bar{p}_{k}^{(\eta)}(s) d s} d \tau .
\end{aligned}
$$

Therefore, the following estimate is true:

$$
\left|\bar{q}_{k}^{(1)}(t)-\bar{q}_{k}^{(0)}(t)\right| \leq \gamma+\left(\gamma+\lambda_{k} \beta|\mu|\right) e .
$$


Given this, we get

$$
\left|\bar{q}_{k}^{(v)}-\bar{q}_{k}\right| \leq \frac{\alpha^{\nu}\left|\bar{q}_{k}^{(1)}-\bar{q}_{k}^{(0)}\right|}{1-\alpha} \leq \frac{\alpha^{\nu}\left(\gamma+\left(\gamma+\lambda_{k} \beta|\mu|\right) e\right)}{1-\alpha}, k=\overline{1, n}, \quad t \in \mathbb{R} .
$$

So, we can write an approximate solution for the equation (2.6) in a form (2.11) by finding an approximation for each of $\bar{p}_{k}(t)$ and $\bar{q}_{k}(t)$.

Thus, the solution of the problem (2.1), (2.2) takes the form

$$
u(x, t)=\sum_{k=1}^{n}\left(c_{k} e^{-\int_{0}^{t} \bar{p}_{k}(s) d s}+\int_{0}^{t} \bar{q}_{k}(\tau) e^{-\int_{\tau}^{t} \bar{p}_{k}(s) d s} d \tau\right) \sin \frac{k \pi x}{l} .
$$

\section{CONCLUSIONS}

So, in our research we found conditions under which there is a global solution of the problem (2.1), (2.2) and constructed it.

\section{REFERENCES}

[1] J. Banaś and I. J. Cabrera, "On solutions of a neutral differential equation with deviating argument." Math. Comput. Modelling, vol. 44, no. 11-12, pp. 1080-1088, 2006, doi: 10.1016/j.mcm.2006.03.012.

[2] M. E. Drakhlin and T. K. Plyshevskaya, "Randwertproblem für eine nichtlineare Differentialgleichung neutralen Typs.” Differ. Uravn., vol. 11, pp. 986-996, 1975.

[3] W. G. El-Sayed, "Solvability of a neutral differential equation with deviated argument." J. Math. Anal. Appl., vol. 327, no. 1, pp. 342-350, 2007, doi: 10.1016/j.jmaa.2006.04.023.

[4] G. A. Kamenskij, A. D. Myshkis, and A. L. Skubachevskij, "Smooth solutions of a boundary value problem for a differential- difference equation of neutral type." Ukr. Math. J., vol. 37, pp. 469-475, 1985, doi: 10.1007/BF01061169.

[5] N. G. Kazakova and D. D. Bajnov, "Ein Randwertproblem für Funktional-Differentialgleichungen neutralen Typs." Math. Slovaca, vol. 30, pp. 105-112, 1980.

[6] D. Y. Khusainov, J. Diblik, and O. I. Ku'zmich, "Convergence estimations of solutions of linear neutral equation.” Din. Sist., Simferopol', vol. 21, pp. 43-53, 2006.

[7] O. I. Kiun, "A boundary value problem for a system of equations of neutral type." Proceedings of MIHM. Automation of chemical industries on the basis of mathematical modeling., vol. 64, pp. $8-11,1975$.

[8] L. A. Minazhdinova, "Continuous dependence on the parameters of a solution of a neutral type equation in Lebesgue spaces." Vestn. Yuzhno-Ural. Gos. Univ., Ser. Mat., Mekh., Fiz., vol. 10, no. 7, pp. 13-18, 2008.

[9] A. E. Rodkina, "Continuability, uniqueness, and continuous dependence on a parameter of solutions of a neutral-type system." Differ. Equations, vol. 11, pp. 204-212, 1976.

[10] A. M. Samoilenko, "On a problem of studying global solutions of linear differential equations with deviating argument." Ukr. Mat. Zh., vol. 55, no. 5, pp. 631-640, 2003.

[11] A. M. Samoilenko and L. M. Serheeva, "Construction of global solutions of partial differential equations with deviating arguments in the time variable." J. Math. Sci., New York, vol. 212, no. 4, pp. 426-441, 2016, doi: 10.1007/s10958-015-2674-0.

[12] L. M. Sergeeva, "Global solutions of neutral differential equations with argument deviation." Scientific Bulletin of Chernivtsi University, Mathematics, vol. 1, no. 3, pp. 108-112, 2011. 
[13] V. P. Skripnik, "Equations with transformed arguments of neutral and superneutral type." Ukr. Math. J., vol. 22, pp. 523-533, 1971, doi: 10.1007/BF01086520.

[14] S. K. Vanani and A. Aminataei, "Multiquadric approximation scheme on the numerical solution of delay differential systems of neutral type." Math. Comput. Modelling, vol. 49, no. 1-2, pp. 234-241, 2009, doi: 10.1016/j.mcm.2008.03.016.

[15] L. A. Zivotovskii, "Zur Frage der Existenz der Lösungen von Differentialgleichungen mit abweichendem Argument neutralen Typs.” Differ. Uravn., vol. 8, pp. 1936-1942, 1972.

[16] A. M. Zverkin, "The existence and uniqueness theorems for equation with a deviating argument in the critical case." Proceedings of the seminar on the theory of differential equations with deviating argument, no. 1, pp. 37-46, 1962.

Authors' addresses

Anatoly M. Samoilenko

Institute of Mathematics of NASU, Differential Equations and Oscillation Theory Department, 3 Tereschenkivska Str., 01004 Kyiv, Ukraine

E-mail address: sameimath.kiev.ua

Lidiya M. Sergeeva

Yuriy Fedkovich Chernivtsi National University, Department of Applied Mathematics, 28 University Str., 58000 Chernivtsi, Ukraine

E-mail address: sergeevalms@gmail.com 